\title{
Sequential CT arterioportography- arteriosplenography depicts individual haemodynamic changes in children with portal hypertension without cirrhosis
}

Simone Hammer ${ }^{1}$, Hans Jürgen Schlitt ${ }^{2}$, Birgit Knoppke ${ }^{3}$, Veronika Ingrid Huf ${ }^{1}$, Walter Alexander Wohlgemuth ${ }^{4}$ and Wibke Uller ${ }^{1 *}$ (D)

\begin{abstract}
We evaluated sequential computed tomography (CT) arterioportography-arteriosplenography for the assessment of venous pathways in children with portal hypertension without cirrhosis. Institutional Review Board approval was obtained for this retrospective, single-centre study. CT was performed after contrast application via catheters placed in the superior mesenteric artery (CT arterioportography) and the splenic artery (CT arteriosplenography) consecutively. Venous pathways in 22 children were evaluated. In all patients, the detailed haemodynamic consequences of portal hypertension could be characterised. The supply of varices at different locations could be assigned to the superior mesenteric vein or splenic vein system. Retrograde blood flow through the splenic vein and inferior mesenteric vein, portosystemic shunting, and patency of splanchnic veins were determined. CT arterioportography-arteriosplenography allowed a complete evaluation of individual haemodynamic pathways in children with portal hypertension.
\end{abstract}

Keywords: Child, Hypertension (portal), Radiology (interventional), Portosystemic shunt (surgical), Tomography (xray computed)

\section{Key points}

- Computed tomography (CT) arterioportography was combined with CT arteriosplenography in one session.

- This technique showed a comprehensive delineation of abdominal venous changes including source of varices, direction of blood flow, and portosystemic shunts.

- Using this approach, planning of individual interventional or surgical management of children with portal hypertension may become more appropriate.

\footnotetext{
* Correspondence: wibke.uller@ukr.de

1 Department of Radiology, University Hospital Regensburg,

Franz-Josef-Strauss-Allee 11, 93053 Regensburg, Germany

Full list of author information is available at the end of the article
}

\section{Background}

Portal hypertension $(\mathrm{PH})$ results from increased pressure in the portal venous system. It is a frequent problem in adult patients with liver cirrhosis. In children, $\mathrm{PH}$ can develop from a variety of conditions, but is frequently associated with intra- or extrahepatic venous obstruction [1]. Consequences of $\mathrm{PH}$ in children are haemodynamic changes in veins in the entire abdomen that include portosystemic shunts, reversal of flow directions, and varices that can lead to gastrointestinal bleeding and, among others, to splenomegaly and thrombocytopenia, ascites, hepatic encephalopathy, and growth failure [2]. Accordingly, morbidity and mortality rates for paediatric $\mathrm{PH}$ are clinically relevant with gastrointestinal bleeding representing the major life-threatening complication [24]. Prevention and treatment of variceal bleeding 
includes pharmacological management, endoscopic, or radiologic interventions and shunt surgery but longlasting therapeutical success is difficult to achieve $[5,6]$.

One major problem for therapy of $\mathrm{PH}$ in children is the high variability of venous pathways in individual patients [7]. The individual assessment of venous circulation in paediatric $\mathrm{PH}$, however, is critical for an adequate therapeutic intervention. To date, no imaging modality has been available to detect portosystemic shunts including the direction of blood flow in all splanchnic veins as well as main supply of varices via the splenic vein (SV) or portal vein (PV) system in every abdominal location simultaneously.

We report herein a new method for the detection of haemodynamic venous changes in paediatric $\mathrm{PH}$. It is based on computed tomography (CT) with sequential contrast application into the splenic artery (SA) and the superior mesenteric artery (SMA). This technique was refined in order to reduce any dilution of contrast media in the systemic circulation and to identify all abdominal venous haemodynamic changes of (1) the superior mesenteric vein (SMV) and (2) the SV system, separately, in particular for differentiating the main supply of varices and identifying blood flow direction.

The objective of this study was to determine feasibility of this innovative technique and to determine the ability to detect individual haemodynamic changes in a small sample of children with portal hypertension.

\section{Methods}

This study was conducted according to the principles expressed in the Declaration of Helsinki. Institutional Review Board approval was obtained. This was a retrospective, single-centre case series of paediatric patients who underwent CT AP-AS at our tertiary referral university hospital centre between November 2011 and September 2018. Children (18 years old or younger) with $\mathrm{PH}$ who underwent CT AP-AS were included.

\section{CT AP-AS technique}

The procedures were performed under general or local anaesthesia and intravenous sedation using a prospectively determined standard angiographic technique. Every patient received $100 \mathrm{IU}$ of heparin per kilogramme body weight at the beginning of the intervention.

The SMA and SA were selectively catheterised by transfemoral approaches using 4-F catheters through 4-F sheaths. Catheters were advanced in the main branch of the SMA and the SA proximal to the first side branch. Two standard digital subtraction angiography studies were performed by separated manual contrast agent injection (Iomeprol; Imeron 300, $300 \mathrm{mg} \mathrm{I/mL,} \mathrm{Bracco} \mathrm{Im-}$ aging SpA, Milan, Italy) in the SA and in the SMA. This was performed (1) to confirm the catheter tip position and (2) to determine the individual time to contrast appearance in the SV and PV or related collateral vessels. This individual time was measured starting from contrast application in the SMA/SA to appearance of contrast medium in the PV/SV using a timer. Afterwards, the patients were transferred to the CT suite with the catheters secured to the skin at the groin.

Sequential CT AP-AS was performed with a helical $256(2 \times 128)$-slice dual source CT scanner (Somatom Flash scanner; Siemens, Forchheim, Germany) using a prospectively determined CT protocol with automatic tube voltage selection $(80 \mathrm{kV}$ or $100 \mathrm{kV})$ and automatic tube current modulation in caudocranial scan direction. First, $0.4 \mathrm{~mL}$ nonionic contrast agent (Iopromid; Ultravist $300,300 \mathrm{mg} \mathrm{I} / \mathrm{mL}$, Bayer Healthcare, Berlin, Germany) was injected per kilogramme bodyweight diluted with the same volume of $\mathrm{NaCl}$ at a rate of $2.5 \mathrm{~mL} /$ $\mathrm{s}$ in the SMA. Scanning was initiated after that time period that was individually measured angiographically, with a CT AP scan delay equal to the time from contrast application in the SMA to appearance in the PV or collateral vessels in the angiogram plus $5 \mathrm{~s}$ due to the rate of contrast agent administration, to achieve maximal contrast appearance in the targeted veins. Then, the same procedure was analogously performed with contrast injection in the SA, with a CT AS scan delay equal to time from contrast application in the SA to appearance in the SV in the angiogram plus $5 \mathrm{~s}$. For image analysis, transverse and coronal plane images with a section thickness of $2.0 \mathrm{~mm}$ and a section increment of $2.0 \mathrm{~mm}$ were reconstructed.

The CT AP-AS images of the 22 patients were evaluated in random order by two radiologists (with 12 and 14 years of experience in the interpretation of vascular CT studies) using a structured template by comparing the enhancement patterns of CT AP and AS, in consensus. Existence and site of varices and differentiation of their origin and main supply (SV system or SMV system) were documented. The presence and localisation of spontaneous portosystemic shunts were distinguished. Intra- and extrahepatic portal vein branches, SMV, and SV were reviewed for patency. Additionally, flow direction in the SV and inferior mesenteric vein (IMV) was determined by comparing the scans performed after contrast injection in the SMA and the SA in every patient. Retrograde blood flow in the SV and IMV was judged to be present in the case of enhancement of these veins after contrast injection into the SMA (Fig. 1a). Available standard $\mathrm{CT}$ in portal venous phase (performed less than 12 months before or after CT AP-AS without any $\mathrm{PH}$ treatment during this period) was compared to CT AP-AS using the same structured template. Readers were blinded to patients' history and clinical data. Effective dose value was calculated by the vendor- 

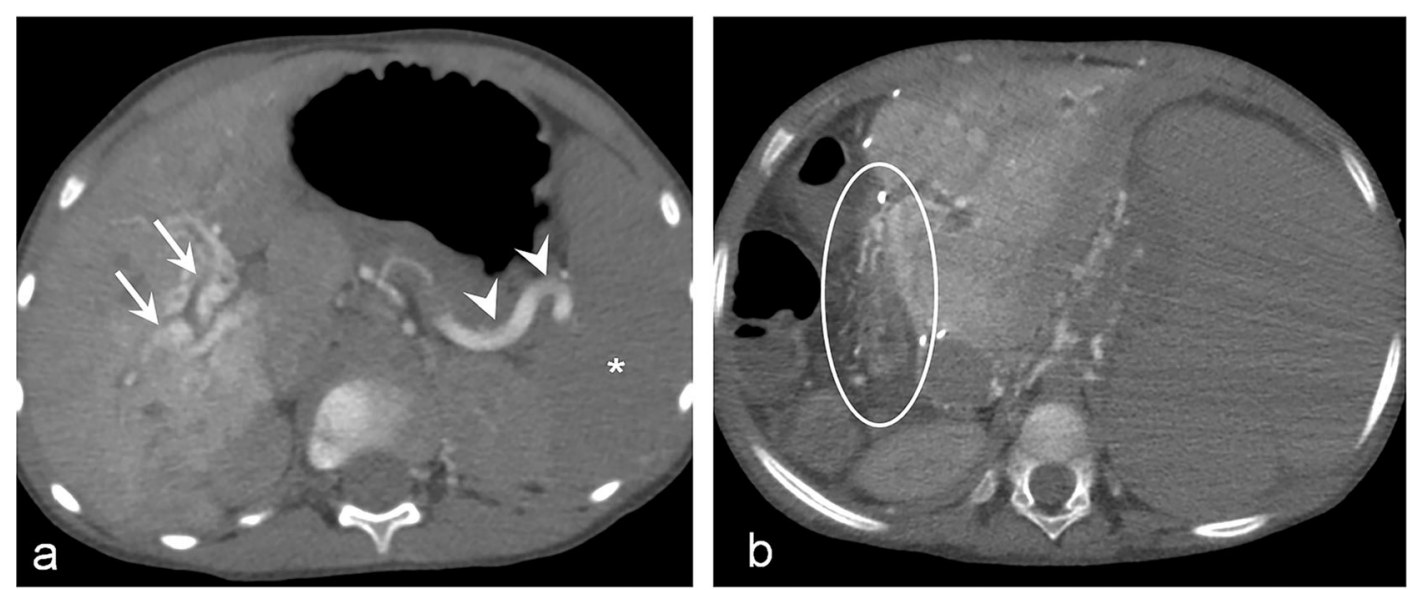

Fig. 1 Computed tomography arterioportography (CT AP) findings in two different children. a A 5-year-old male patient with portal vein thrombosis. Axial images obtained after contrast injection in the superior mesenteric artery detect portal biliopathy (arrows) and reversed blood flow in the splenic vein (arrowheads). The spleen does not show contrast enhancement (white asterisk). b An 8-year-old male patient with portal vein thrombosis after liver transplantation. CT AP shows varices and diffuse enhancement of the hepaticojejunostomy (ellipse)

specific software Teamplay Dose (Siemens, Forchheim, Germany). Numerical variables were summarised using descriptive statistics (median, minimum, and maximum). Categorical variables were reported as frequency and percentage.

\section{Results}

Between November 2011 and September 2018, 25 children underwent CT AP-AS. Three patients without diagnosis of $\mathrm{PH}$ were excluded. Accordingly, the patient population consisted of 22 children with $\mathrm{PH}$ without cirrhosis who underwent CT AP-AS (median age 8.6 years, $2.6-17.4$ years; median body weight $29.4 \mathrm{~kg}, 14.0-79.7$ $\mathrm{kg})$.

Fifteen children suffered from extrahepatic portal vein obstruction. The PH resulted in splenomegaly $(n=21)$, hypersplenism $(n=18)$, status post-gastrointestinal bleeding $(n=12)$, additional anaemia $(n=11)$, and ascites $(n=5)$. CT AP-AS was performed with technical success in all cases. The median scan delay was $20 \mathrm{~s}$ (14-28 s) for CT AP and 18 s (12-29s) for CT AS, respectively. Median dose-length product for both CT scans was $338 \mathrm{mGy} \times \mathrm{cm}(49-1128 \mathrm{mGy} \times \mathrm{cm})$. Median effective dose value was $7.5 \mathrm{mSv}(1.1-17.1 \mathrm{mSv})$. No complications related to this technique occurred.

Oesophageal $(n=16)$, paraoesophageal $(n=18)$, gastric $(n=21)$, duodenal $(n=11)$, small intestinal $(n=11)$, colonic $(n=9)$, rectal $(n=6)$, peripancreatic $(n=7)$, umbilical $(n=2)$, and paraumbilical $(n=2)$ varices were detected. Varices located at the hepaticojejunostomy were detected in 3 of 4 of children who previously underwent liver transplantation (Fig. 1b). Peribiliar $(n=$ $18)$ and gallbladder varices $(n=9)$ were identified.
All varices located within the small intestine, hepaticojejunostomy, colon, and gallbladder were supplied by the SMV. The following varices were supplied by the SMV system, the SV system, or both (Fig. 2): oesophageal (6 SMV, 7 SV, 3 both), paraoesophageal (5 SMV, 12 SV, 1 both), gastric (7 SMV, 14 SV), duodenal (7 SMV, $3 \mathrm{SV}, 1$ both), peripancreatic (2 SMV, $2 \mathrm{SV}, 3$ both), and rectal varices (2 SMV, $4 \mathrm{SV})$.

Obstruction of the SMV $(n=2)$ and SV $(n=1)$ was found. The SMV-SV confluence was occluded in 6 cases. Occlusion of the extrahepatic PV $(n=15)$ and intrahepatic PV $(n=11)$ was detected resulting in extra- and intrahepatic cavernous transformation of the PV in 15 and 5 cases, respectively. Additionally, 16 children showed signs of portal biliopathy (multiple extrinsic/ peribiliary collateral vessels, irregular and dilated bile ducts). Retrograde venous blood flow was seen in the SV in 14 cases and in the IMV in 8 cases. Splenorenal $(n=$ 20 ) and additional shunts (vena haemiazygos, $n=10$; inferior caval vein, $n=7$ ) were found. Table 1 summarises the findings of CT AP-AS.

In 5 cases, standard $\mathrm{CT}$ in portal venous phase was available. Oesophageal varices were detected in both, CT in portal venous phase and CT AP-AS equally. Of these 5 patients, CT in portal venous phase did not show any gastric, duodenal, small intestine, and colonic varices while CT AP-AS detected gastric varices in 5/5, duodenal varices in $2 / 5$, small intestine varices in $3 / 5$, and colonic varices in $3 / 5$ patients (Fig. 3). Furthermore, CT AP-AS showed signs of portal biliopathy in 3 cases and a total of 10 portosystemic shunts, whereas none of these changes was detected by standard CT in portal venous phase. Extrahepatic and intrahepatic PV were occluded on standard CT in 2 and 3 cases, respectively, 

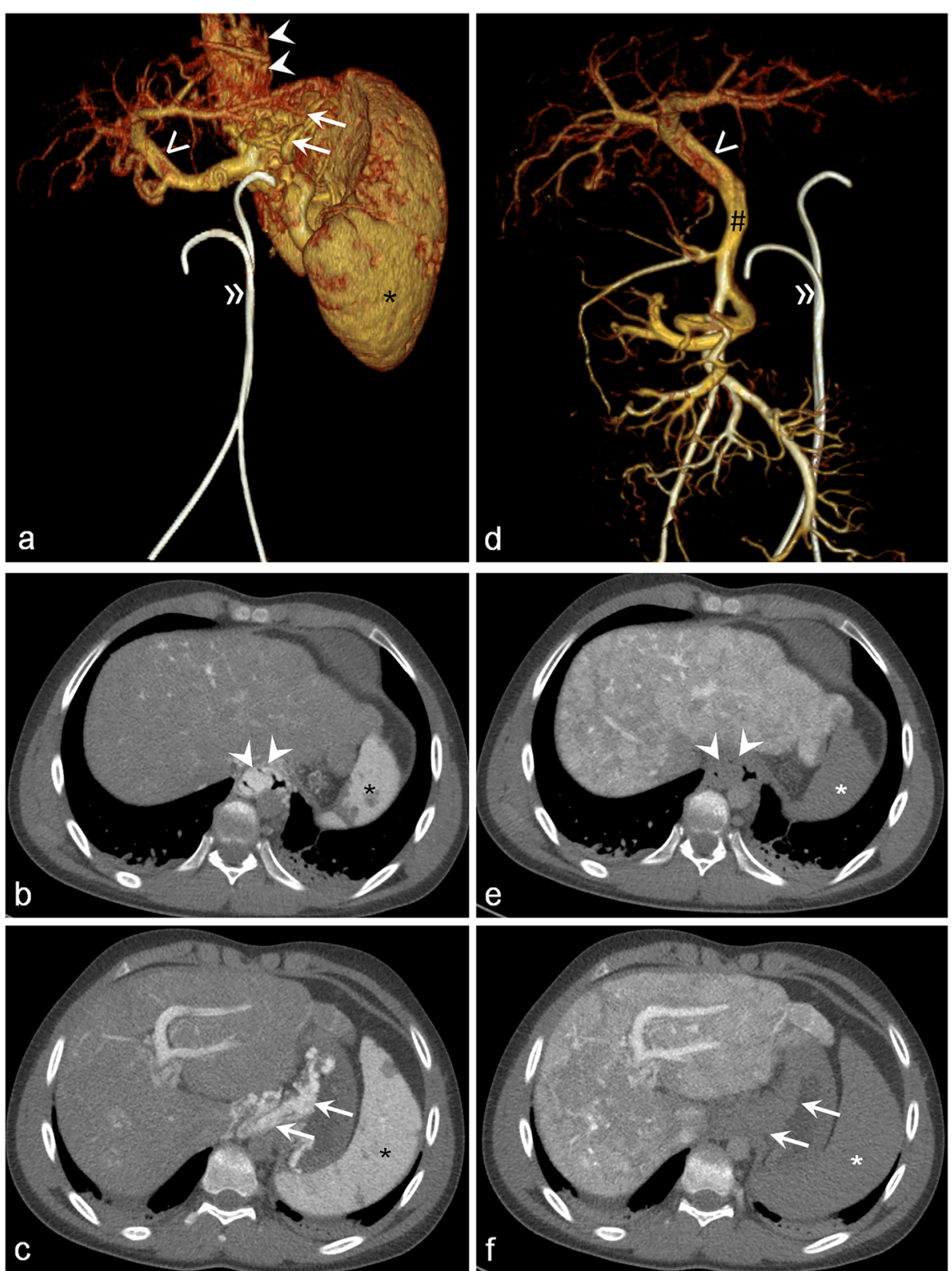

Fig. 2 A 16-year-old male patient with portal hypertension. a-c Volume rendering and axial images of computed tomography arteriosplenography after contrast injection into the splenic artery allow to detect filling of oesophageal (b, arrowheads) and gastric (c, arrows) varices. Note the contrast enhancement of the spleen (black asterisk). $\mathbf{d}-\mathbf{f}$ Volume rendering and axial images of computed tomography arterioportography after contrast injection into the superior mesenteric artery detect filling of the superior mesenteric vein (hashtag) and portal vein (open arrowhead) without any filling of oesophageal (e, arrowheads) or gastric varices (f, arrows). Note the absence of contrast enhancement of the spleen (white asterisk). Catheters placed in the superior mesenteric and splenic artery (double arrow). Portal vein (open arrowhead). Superior mesenteric vein (hashtag)

while CT AP-AS revealed patency of the extrahepatic and intrahepatic PV in these patients.

\section{Discussion}

CT AP with contrast application in the SMA was initially performed to detect liver lesions [8-12]. Reinig et al. [13] used this technique for evaluating the anatomy of the PV system and the extent of PV thrombosis to facilitate accurate anatomical assessment prior to shunt surgery. This approach was also used for more accurate delineation of portosystemic shunts [14, 15]. However, contrast injection solely into the SMA has never been combined with CT AS to evaluate varices and their main venous supply as well as reversal blood flow, portosystemic shunts, and patency of splanchnic veins. 
Table 1 Haemodynamic changes in 22 children with portal hypertension detected by sequential computed tomography arterioportography-arteriosplenography

\begin{tabular}{|c|c|c|c|c|}
\hline \multirow[t]{2}{*}{ Findings } & \multirow{2}{*}{$\begin{array}{l}\text { Number } \\
\text { of } \\
\text { findings }\end{array}$} & \multicolumn{3}{|c|}{ Main venous supply of varices by } \\
\hline & & SMV & SV & SMV and SV \\
\hline \multicolumn{5}{|l|}{ Varices } \\
\hline Oesophageal & 16 & 6 & 7 & 3 \\
\hline Paraoesophageal & 18 & 5 & 12 & 1 \\
\hline Gastric & 21 & 7 & 14 & 0 \\
\hline Duodenal & 11 & 7 & 3 & 1 \\
\hline Small intestinal & 11 & 11 & 0 & 0 \\
\hline Colonic & 9 & 9 & 0 & 0 \\
\hline Rectal & 6 & 2 & 4 & 0 \\
\hline Peripancreatic & 7 & 2 & 2 & 3 \\
\hline Umbilical & 2 & 2 & 0 & 0 \\
\hline Paraumbilical & 2 & 0 & 0 & 2 \\
\hline Biliary & 18 & 18 & 0 & 0 \\
\hline Portal biliopathy & 16 & & & \\
\hline Gallbladder & 9 & 9 & 0 & 0 \\
\hline Hepaticojejunostomy & 3 & 3 & 0 & 0 \\
\hline \multicolumn{5}{|l|}{ Portosystemic shunts } \\
\hline Splenorenal & 20 & & & \\
\hline Shunt to haemiazygos & 10 & & & \\
\hline Shunt to inferior caval vein & 7 & & & \\
\hline \multicolumn{5}{|l|}{ Occlusion of splanchnic veins } \\
\hline Extrahepatic portal vein & 15 & & & \\
\hline Cavernous transformation & 15 & & & \\
\hline \multicolumn{5}{|l|}{ Extrahepatic portal vein } \\
\hline Intrahepatic portal vein & 11 & & & \\
\hline Cavernous transformation & 5 & & & \\
\hline \multicolumn{5}{|l|}{ Intrahepatic portal vein } \\
\hline Extra- and intrahepatic portal vein & 10 & & & \\
\hline SMV & 2 & & & \\
\hline SV & 1 & & & \\
\hline Confluence & 6 & & & \\
\hline \multicolumn{5}{|l|}{ Retrograde venous blood flow } \\
\hline SV & 14 & & & \\
\hline Inferior mesenteric vein & 8 & & & \\
\hline
\end{tabular}

SMV Superior mesenteric vein, SV Splenic vein

Using combined CT AP-AS, we were able to demonstrate a wide range of variable individual haemodynamic changes in children with portal hypertension. Venous routes and different tributaries varied considerably. Interestingly, the source of varices differed extremely and could be assigned to the SV or the SMV system. Additionally, even small varices at the hepaticojejunostomy in children after liver transplantation were identified as source of occult bleeding.
Hence, this technique was also able to identify varices in localisations where endoscopy is not easily feasible or fails $[16,17]$. Furthermore, our technique detected occlusion of splanchnic veins or changes in blood flow direction. Although the flow of the splanchnic veins can in principle also be detected using ultrasonography, in our experience, overlying structures often interfere with direct imaging of the SMV or SV in paediatric ultrasonography. 

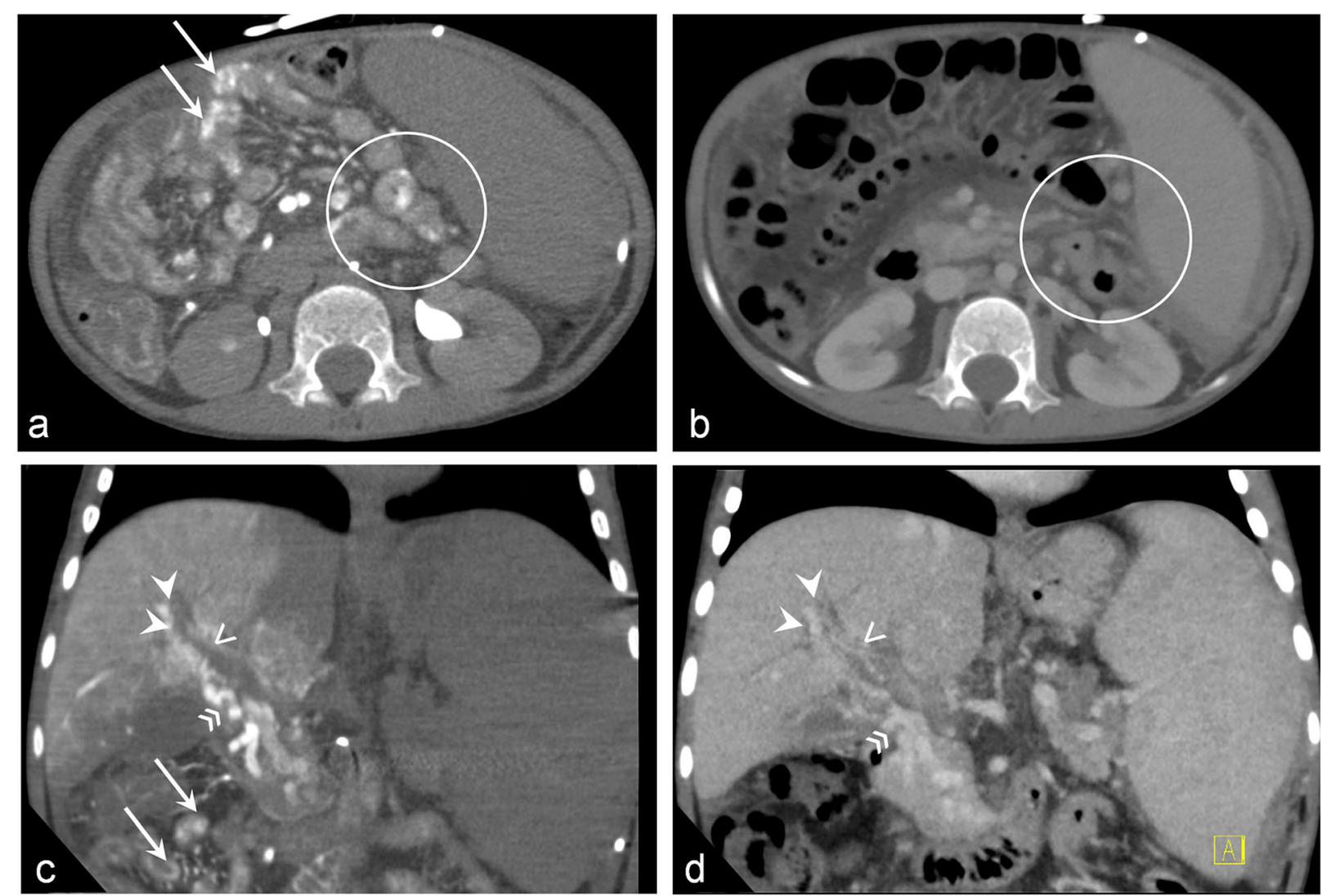

Fig. 3 Comparison of computed tomography (CT) arterioportography (AP) $(\mathbf{a}, \mathbf{c})$ with standard $C T$ after intravenous contrast injection (portal venous phase: $\mathbf{b}, \mathbf{d}$ ) in a 6-year-old male patient with portal vein thrombosis. Axial (a) and coronal (c) images of CT AP after contrast injection into the superior mesenteric artery allow to clearly detect multiple jejunal varices (arrows and circle) and portal biliopathy (arrowheads and open arrows: multiple small peribiliary collaterals, irregular and dilated bile ducts). Axial (b) and coronal (d) standard CT in the portal venous phase after intravenous contrast injection performed 2 weeks before CT AP does not detect any jejunal varices; evidence of portal biliopathy is lower than that provided by CT AP

Following direct contrast injection into the SA and the SMA, no dilution effects from the systemic circulation occurred. This is critical for obtaining a strong contrast enhancement in the splanchnic veins and varices, allowing accurate anatomical and dynamical assessment. Compared to standard contrast-enhanced CT in portal venous phase, CT AP-AS proved to be superior not only in detecting the source of varices but also in the detection of different varices in other localisations than oesophageal. According to our results, CT AP-AS also proved patency of the extra- and intrahepatic PV, although standard contrast-enhanced CT did not show contrast enhancement in these vessels and simulated occlusions.

To our knowledge, combined CT AS-AP is the first technique that reveals every kind of haemodynamic venous changes in all abdominal locations in an objective, threedimensional way and allows multiplanar reformatting. This comprehensive delineation of venous haemodynamic changes is of particular interest in complicated cases where the most appropriate management approach needs to be discussed and determined by interdisciplinary teams of interventional radiologists, surgeons, and gastroenterologists.

The major limitations of our study are that we did not have any comparative control group available and comparative imaging techniques as reference standard are missing.

In conclusion, although this study is additionally limited by its retrospective design and the small study cohort (based on the low incidence of $\mathrm{PH}$ without cirrhosis in children), our approach can help in detecting changes with consequences for individual treatment decisions. Important clinical points are as follows: (1) portosystemic shunts not visible on standard CT angiography may be a source of alternative access routes for interventional radiological treatment of varices (e.g., via the femoral vein/renal vein/inferior of superior caval vein), (2) delineation of patency and reversal flow in the splanchnic veins is of particular interest prior to shunt surgery, and (3) the individual source of varices is of 
utmost interest in planning decompression of either the SV or the SMV system. No imaging modality was available to answer all these relevant questions in symptomatic paediatric $\mathrm{PH}$ simultaneously. However, all this information needs to be available in order to plan and adapt further management in every single case since indiscriminate interventions may result in deterioration of PH. CT AP-AS could be applied before more invasive procedures are considered. Future studies are necessary to address treatment outcome of individual management of complicated $\mathrm{PH}$ in children based on CT AP-AS findings.

\section{Abbreviations}

AP: Arterioportography; AS: Arteriosplenography; CT: Computed tomography; IMV: Inferior mesenteric vein; PH: Portal hypertension; PV: Portal vein;

SA: Splenic artery; SMA: Superior mesenteric artery; SMV: Superior mesenteric vein; SV: Splenic vein

\section{Authors' contributions}

SH and WU contributed to the conceptualisation/design of the study, analysed the data, and wrote the manuscript. All authors contributed to the revision of the manuscript and approved the final manuscript.

\section{Funding}

The authors state that this work has not received any funding. Open Access funding enabled and organised by Projekt DEAL.

\section{Availability of data and materials}

All relevant data is available within the manuscript.

\section{Ethics approval and consent to participate}

Institutional Research Ethics Board approval was obtained (University of Regensburg). Individual consent for inclusion in the review was waived by the Research Ethics Board.

\section{Consent for publication}

Not applicable.

\section{Competing interests}

The authors declare that they have no competing interests.

\section{Author details}

'Department of Radiology, University Hospital Regensburg, Franz-Josef-Strauss-Allee 11, 93053 Regensburg, Germany. ${ }^{2}$ Department of Surgery, University Hospital Regensburg, Franz-Josef-Strauss-Allee 11, 93053 Regensburg, Germany. ${ }^{3} \mathrm{KUNO}$ University Children's Hospital, University Hospital Regensburg, Franz-Josef-Strauss-Allee 11, 93053 Regensburg, Germany. ${ }^{4}$ Department of Radiology, University Hospital Halle, Ernst-Grube-Str. 40, 06120 Halle, Germany.

Received: 18 August 2020 Accepted: 4 November 2020

Published online: 02 December 2020

\section{References}

1. Vogel CB (2017) Pediatric portal hypertension: a review for primary care. Nurse Pract 42:35-42. https://doi.org/10.1097/01.NPR.0000515427.91649.91

2. Grammatikopoulos T, McKiernan PJ, Dhawan A (2018) Portal hypertension and its management in children. Arch Dis Child 103:186-191. https://doi. org/10.1136/archdischild-2015-310022

3. Shneider B, Emre S, Groszmann R et al (2006) Expert pediatric opinion on the Report of the Baveno IV consensus workshop on methodology of diagnosis and therapy in portal hypertension. Pediatr Transplant 10:893-907. https://doi.org/10.1111/j.1399-3046.2006.00597.x

4. Shneider BL, Bosch J, de Franchis R et al (2012) Portal hypertension in children: expert pediatric opinion on the report of the Baveno v Consensus Workshop on Methodology of Diagnosis and Therapy in Portal
Hypertension. Pediatr Transplant 16:426-437. https://doi.org/10.1111/j.13993046.2012.01652.x

5. Cortez AR, Kassam A-F, Jenkins TM et al (2019) The role of surgical shunts in the treatment of pediatric portal hypertension. Surgery 166:907-913. https:// doi.org/10.1016/j.surg.2019.05.009

6. Kassam A-F, Goddard GR, Johnston ME et al (2020) Natural course of pediatric portal hypertension. Hepatol Commun 4:1346-1352. https://doi. org/10.1002/hep4.1560

7. Kirby JM, Cho KJ, Midia M (2013) Image-guided intervention in management of complications of portal hypertension: more than TIPS for success. Radiographics 33:1473-1496. https://doi.org/10.1148/rg.335125166

8. Ferrucci JT (1990) Liver tumor imaging: current concepts. AJR Am J Roentgenol 155:473-484. https://doi.org/10.2214/ajr.155.3.2117343

9. Lupetin AR, Cammisa BA, Beckman I et al (1996) Spiral CT during arterial portography. Radiographics 16:723-743; discussion 743-6. https://doi.org/10. 1148/radiographics.16.4.8835967

10. Matsui O, Takashima T, Kadoya M et al (1985) Dynamic computed tomography during arterial portography: the most sensitive examination for small hepatocellular carcinomas. J Comput Assist Tomogr 9:19-24. https:// doi.org/10.1097/00004728-198501000-00004

11. Matsui O, Takashima T, Kadoya M et al (1987) Liver metastases from colorectal cancers: detection with $\mathrm{CT}$ during arterial portography. Radiology 165:65-69. https://doi.org/10.1148/radiology.165.1.2819942

12. Soyer P, Bluemke DA, Fishman EK (1994) CT during arterial portography for the preoperative evaluation of hepatic tumors: how, when, and why? AJR Am J Roentgenol 163:1325-1331. https://doi.org/10.2214/ajr.163.6.7992722

13. Reinig JW, Sanchez FW, Vujic I (1985) Hemodynamics of portal blood flow shown by CT portography. Work in progress. Radiology 154:473-476. https://doi.org/10.1148/radiology.154.2.3966135

14. Ibukuro K, Tsukiyama T, Mori K, Inoue Y (1998) Veins of Retzius at CT during arterial portography: anatomy and clinical importance. Radiology 209:793800. https://doi.org/10.1148/radiology.209.3.9844676

15. Terayama N, Matsui O, Kobayashi S et al (2008) Portosystemic shunt on CT during arterial portography: prevalence in patients with and without liver cirrhosis. Abdom Imaging 33:80-86. https://doi.org/10.1007/s00261-0079196-2

16. Nishimura N, Yamamoto H, Yano T et al (2010) Safety and efficacy of double-balloon enteroscopy in pediatric patients. Gastrointest Endosc 71: 287-294. https://doi.org/10.1016/j.gie.2009.08.010

17. Sanada Y, Mizuta K, Yano T et al (2011) Double-balloon enteroscopy for bilioenteric anastomotic stricture after pediatric living donor liver transplantation. Transpl Int 24:85-90. https://doi.org/10.1111/j.1432-2277. 2010.01156.x

\section{Publisher's Note}

Springer Nature remains neutral with regard to jurisdictional claims in published maps and institutional affiliations.

\section{Submit your manuscript to a SpringerOpen ${ }^{\circ}$ journal and benefit from:}

- Convenient online submission

- Rigorous peer review

- Open access: articles freely available online

High visibility within the field

- Retaining the copyright to your article

Submit your next manuscript at $\boldsymbol{\nabla}$ springeropen.com 